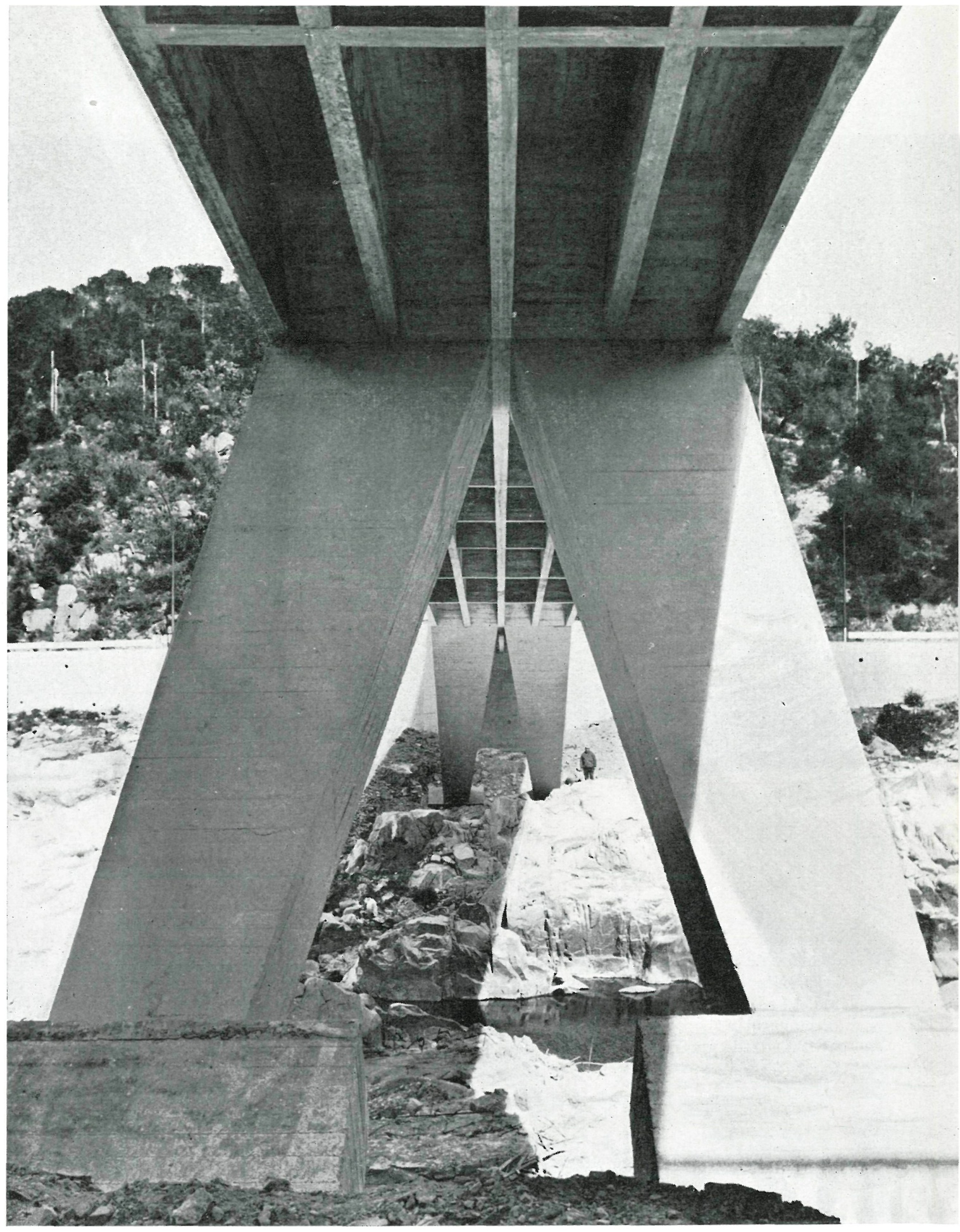

$562-109$

\title{
puente de hormigón pretensado sobre el río Ter
}

\section{ARTURO REBOLLO, Ingeniero de Caminos}

Sim Dpsis Esta obra, construida en 1964, es el acceso utilizado para la construcción, montaje y actualmente para la explotación de la central subterránea del Salto de Susqueda (Gerona), de HIDROELECTRICA DE CATALUNA, S. A.

La estructura, prevista para sobrecargas móviles de $125 \mathrm{t}$, está formada por un pórtico simple con apoyos inclinados y variación lineal en los cantos. El tablero, de $60 \mathrm{~m}$ de longitud, va pretensado de extremo a extremo, presentando articulaciones móviles en los mismos.

En vista del excelente resultado de esta disposición estructural, se concluye con la posibilidad de ampliarla hasta el triple de la escala, pudiéndose salvar así luces libres de $120.140 \mathrm{~m}$, no solamente por permitirlo sus condiciones técnicas y económicas, sino también porque se lograrían puentes de notable belleza con este tipo de estructura. 


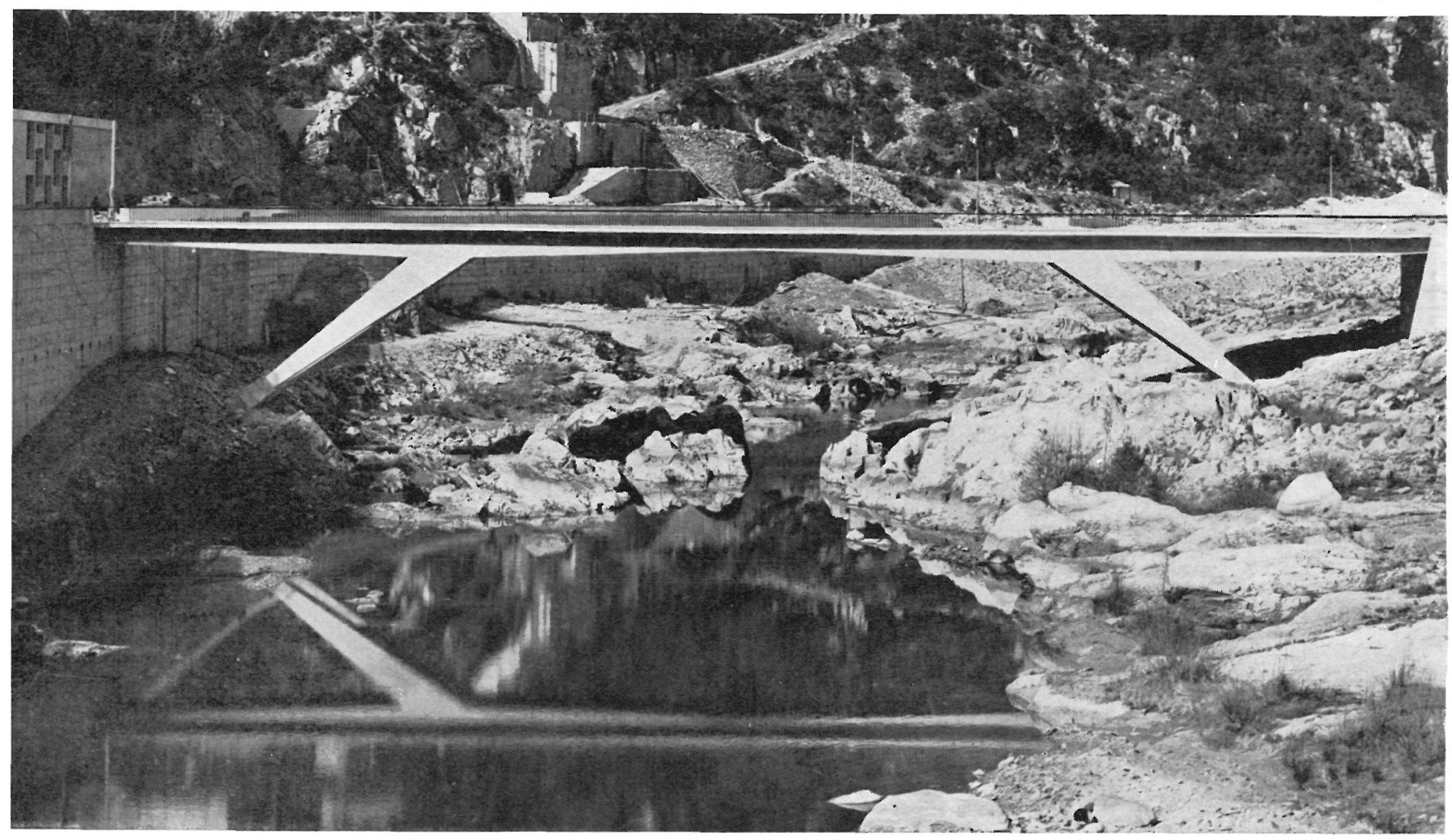

Se trataba de cruzar a la margen derecha del río Ter para iniciar la construcción de la Central subterránea del Salto de Susqueda (Gerona) y, posteriormente, no sólo transportar toda la maquinaria pesada de sus instalaciones, sino también tener acceso a la misma durante su futura explotación. El planteamiento inicial del problema delimitaba claramente la función del puente; sin embargo, el carácter singular del conjunto de la instalación y del paisaje en que estaba encajada nos pareció, desde un principio, que exigía algo más que la realización de una obra simplemente funcional.

\section{El proyecto}

\subsection{La solución elegida}

Fue con esta idea con la que iniciamos los tanteos de diversas soluciones en las que todas tenían por denominador común salvar el cauce, cuya anchura en aquella zona oscilaba cntre los 60 y $70 \mathrm{~m}$, con una obra de una sola luz, bien en tramo recto, bien en arco, llegando finalmente a la solución de transformar a este último en un pórtico simple de apoyos inclinados con variación lineal en los cantos.

A primera vista la solución formal no encuentra quizá una justificación si se tiene en cuenta que bajo este puente discurre un río como el Ter; pero esta situación solamente ha de producirse, una 
vez derivado el río a la Central, cuando tengan lugar los vertidos, siempre esporádicos, de la presa. Por ello, y porque sus líneas, sumamente escuetas, armonizaban perfectamente con la aridez de las rocas del cauce y con la agreste naturaleza del lugar, decidimos elegir una solución de este tipo que además permitía, con amplitud, el desagüe para los mayores caudales que pudieran presentarse en el río.

\subsection{Sus características principales}

Este pórtico de hormigón armado tiene una longitud de tablero de $60 \mathrm{~m}$, formado por cinco vigas longitudinales, arriostradas transversalmente, con cantos comprendidos entre los 0,70 y $1,20 \mathrm{~m}$ sobre los que el forjado determina una anchura de circulación de $5,80 \mathrm{~m}$, suficiente para el caso.

Los apoyos inclinados, dos en cada margen, forman viga-cajón; sus esfuerzos se transmiten a la roca a través de zapatas de repartición, empotradas en la misma. La roca de cimentación presenta buenas condiciones geotécnicas para resistir aquellos esfuerzos, no habiendo sido necesario más que prever un cosido con anclajes normales embutidos en el hormigón. Estos apoyos inclinados determinan una luz libre de $46 \mathrm{~m}$ sobre el lecho del río.

La esbeltez de las líneas del puente ha exigido la aplicación de unas tensiones de pretensado, longitudinales a las vigas, a través de 15 cables de $12 \varnothing 7$ que totalizan aproximadamente un esfuerzo complementario de $750 \mathrm{t}$ nominales. Estos cables cubren toda la longitud del tablero, más de $60 \mathrm{~m}$, presentando dos senos acusados en las zonas de incidencia de los apoyos inclinados, en razón de las leyes de máximos esfuerzos.

Esta disposición longitudinal de pretensado ha condicionado que los apoyos de los extremos del tablero fuesen articulaciones móviles para, de esta forma, absorber cualquier tipo de deformación que pudiera presentarse incluso durante el tesado de los cables.

La obra en sí, aparte de los muros estribo, no cubica más que $200 \mathrm{~m}^{3}$ de hormigón, reforzado entre armaduras y anclajes con $22 \mathrm{t}$ de acero especial corrugado y $3,3 \mathrm{t}$ de acero especial para los cables del tablero, lo que en conjunto da un índice de $125 \mathrm{~kg}$ de acero por $\mathrm{m}^{3}$ de hormigón.

\subsection{El cálculo de la estructura}

Para los efectos de cálculo hemos considerado a la roca como indeformable y el hormigón armado con las siguientes constantes:

\begin{tabular}{|c|c|}
\hline $\begin{array}{llllllllll}\text { peso } & \text { específico } & \ldots & \ldots & \ldots & \ldots & \ldots & \ldots & \ldots & \ldots\end{array}$ & $2,5 \mathrm{t} / \mathrm{m}^{3}$ \\
\hline dilatación térmica $\ldots \ldots \ldots \ldots \ldots \ldots$ & $9 \times 10^{-6} /{ }^{\circ} \mathrm{C}$ \\
\hline 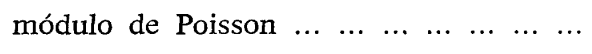 & 0,2 \\
\hline factor de corte $\begin{array}{llllllllll} & \ldots & \ldots & \ldots & \ldots & \ldots & \ldots & \ldots & \ldots & \ldots\end{array}$ & 1,25 \\
\hline módulo de elasticidad & $2 \times 10^{-6} \mathrm{t} / \mathrm{m}^{2}$ \\
\hline
\end{tabular}

Hemos considerado actuando sobre la estructura los siguientes efectos: peso propio; temperatura y retracción equivalente a una disminución de $20^{\circ} \mathrm{C}$; pretensado; y una sobrecarga móvil formada 


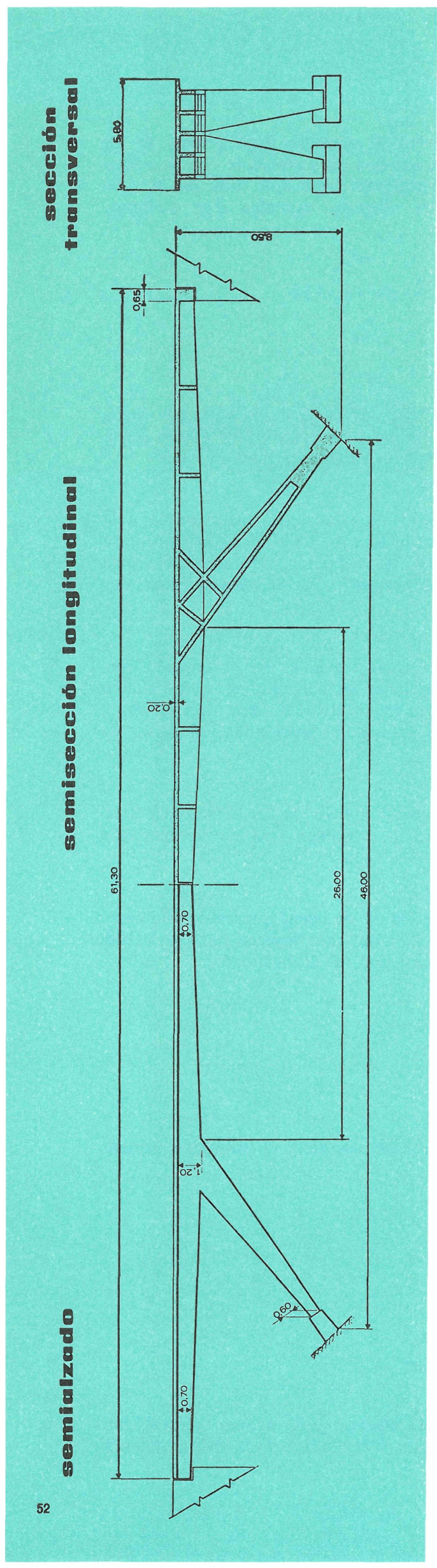

por un vehículo especial de las siguientes características: cuatro ejes distantes 4-5-3-9 $\mathrm{m}$ sobre los que inciden unos pesos muertos de $6,4,10,5 \mathrm{t}$ y una carga de $0,0,40,60 \mathrm{t}$; o sea, un vehículo con un peso total de $125 \mathrm{t}$, sometido además todo él a un impacto dinámico del 25 por 100 .

No hemos considerado los efectos de frenado, viento y repartición transversal de esfuerzos, puesto que no tienen importancia en nuestro caso.

La estructura presentaba, en las diversas hipótesis de carga, cinco reacciones hiperestáticas y para su determinación igualamos en la sección central las deformaciones correspondientes a cada semipórtico, dos desplazamientos y un giro, obligando además a que los desplazamientos verticales en las dos articulaciones móviles de los extremos fuesen nulos; a lo largo del proceso numérico ha sido preciso tener en cuenta la notable variación de la inercia de las secciones del tablero y de las secciones en cajón de los apoyos inclinados. Determinados los esfuerzos le superpusimos el efecto del pretensado, pasando a continuación al dimensionado de las secciones en rotura y suponiendo unas pérdidas por rozamiento y relajación de un 15 por 100 en el rendimiento de los cables.

Este proceso lo repetimos varias veces aquilatando sucesivamente las dimensiones de los cantos y la cuantía de las armaduras. En toda esta ardua tarea colaboró el licenciado en Ciencias Físicas, M. Serra.

\section{Su comstrucceión}

La realizamos por administración directa con los equipos de obra de Hidroeléctrica de Cataluña, S. A., y con la colaboración del ingeniero de Caminos J. M. Sanz.

\subsection{Defalles constructivos}

Cabe señalar que la posibilidad de riadas obligó a construir previamente unas pilas de hormigón pobre en el cauce del río para apoyar sobre ellas la cimbra, a una altura que cubriera lo más posible el riesgo de ser alcanzada por el agua, porque la construcción la tuvimos que fijar inevitablemente para el otoño de 1964, época coincidente con las mayores aportaciones del río.

Como se comprende el mayor trabajo incidió sobre el taller de carpintería. La puesta en obra del hormigón, que duró horas, se realizó primeramente para los dos apoyos inclinados y posteriormente, pasados algunos días, para el tablero, conjuntamente vigas y forjado. La dosificación de cemento fue de $350 \mathrm{~kg} / \mathrm{m}^{3}$. 


\section{sección del tablero}

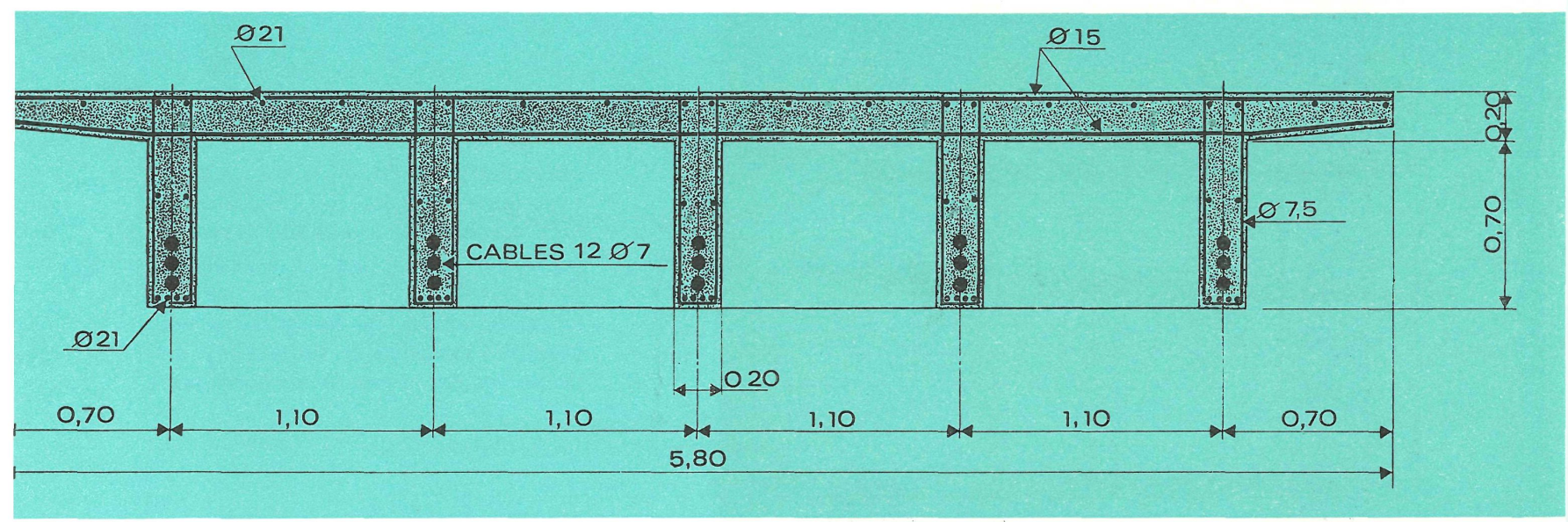

El principal problema que se nos presentó en la obra y que pudo acarrear graves consecuencias, fue motivado por las vainas de los cables. En aquel momento no teníamos ninguna referencia de una operación de tesado similar en nuestro país para cables de más de $60 \mathrm{~m}$ con varias inflexiones, y por otra parte tampoco conocíamos en el mercado vainas adecuadas para nuestro caso; las que se venían usando normalmente en piezas isostáticas hubo finalmente que desecharlas, ya que al ceñirse a las inflexiones de los cables se deformaban y abrían peligrosamente, habiendo ocasionado, sin duda, la inutilización del tesado por alcanzar el hormigón los elementos de los cables. A la vista de la situación optamos por una solución nada ortodoxa, según la opinión de los técnicos, pero que por ahora nos ha dado unos resultados inmejorables, y que consistió en usar sencillamente vainas de plástico. Han presentado - a nuestro modo de ver- tres ventajas: $1 .^{\mathrm{a}}$, que se ciñen perfectamente a cualquier directriz de los cables, incluso para formas helicoidales, según hemos utilizado recientemente; $2 .^{a}$, que las pérdidas por rozamiento son mínimas; hemos comprobado que en piezas de más de $70 \mathrm{~m}$, hiperestáticas también, con un simple golpe de maza los elementos del cable deslizan perfectamente a lo largo de la vaina una vez hormigonada la pieza; y 3. ${ }^{a}$, su precio.

No queremos dejar de mencionar lo anecdótico que, por lo imprevisto, pudo costarle a esta obra su existencia. Cuando el tiempo ya nos apremiaba para poner en servicio el puente y habiendo comenzado las brigadas de turno a verter el primer cubo de hormigón en el tablero, precisamente el 22 de diciembre de 1964, la inesperada fortuna repartió entre aquellos operarios, a través del premio gordo de la lotería, ¡más de 33 millones de pesetas! El hormigón pudo terminarse aquella misma noche, entre la tensión y la duda de dejar abandonado el puente a medio construir. A la mañana siguiente, la mayoría millonarios, todos habían desaparecido.

\subsection{Resulfados}

La puesta en servicio vino a corroborar todas las previsiones. En el año 1966 se presentó con toda solemnidad la góndola especial con los transformadores de la Central, reproduciendo al pie de la letra el tren de cargas analizado en el cálculo, o sea, una sobrecarga móvil aproximada de 120 t. Las diversas observaciones dieron en la clave del pórtico $2 \mathrm{~mm}$ de flecha. No hubo más novedad.

Debemos señalar que, con los medios casi rudimentarios con que contábamos y con los precios de aquel entonces, el coste de la obra, incluyendo los gastos generales, dio un índice de 9.000 pesetas por $\mathrm{m}^{2}$ de tablero, que es para tomar en consideración. Naturalmente, es preciso tener en cuenta que la obra fue realizada por administración directa y que no hubo problemas en la cimentación, 


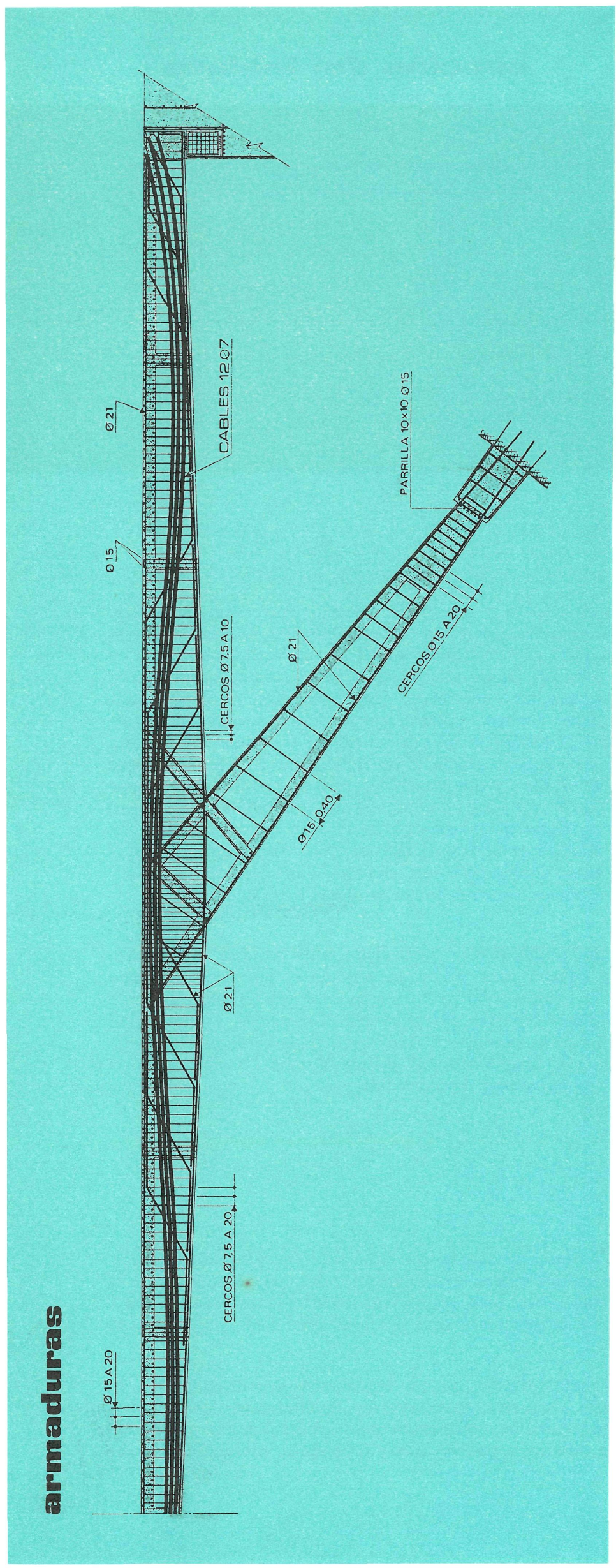

sin embargo, no deja de ser un buen exponente para las posibilidades de esta estructura, quedando bien patente que no obliga ni a costosos procesos de mano de obra, dada la simplicidad de sus líneas, ni a una cantidad excesiva de materiales, dado su escaso volumen de hormigón.

\section{Posibinidades de este thipo de estructetura}

Debemos concluir que el habernos decidido a exponer los detalles de esta obra, aunque con algo de retraso, ha sido con objeto de hacer ver esencialmente en ella una realización a escala reducida de lo que realmente creemos puede dar de sí esta estructura.

\subsection{Posibilidades téenicas y económicas}

Vemos fundamentalmente la posibilidad de llegar a unas dimensiones triples de las expuestas, lo que significaría una longitud de tablero de unos $180 \mathrm{~m}$, que no vemos ninguna dificultad en tensar de extremo a extremo, máxime cuando actualmente en el mercado podemos obtener torones especiales para pretensado con los que lograríamos fácilmente cables de triple rendimiento y potencia, a igualdad de diámetro de vainas; ello nos conduciría a salvar luces libres entre los 120 y $140 \mathrm{~m}$, con anchuras de tablero entre los 12 y $18 \mathrm{~m}$. Uniendo a esta ampliación de escala la posibilidad de proyectar la sección del tablero en cajón, e incluso de reducir a cuatro el número de las vigas principales, se podría jugar con una mayor capacidad resistente y con una mayor simplificación de los elementos del puente. 

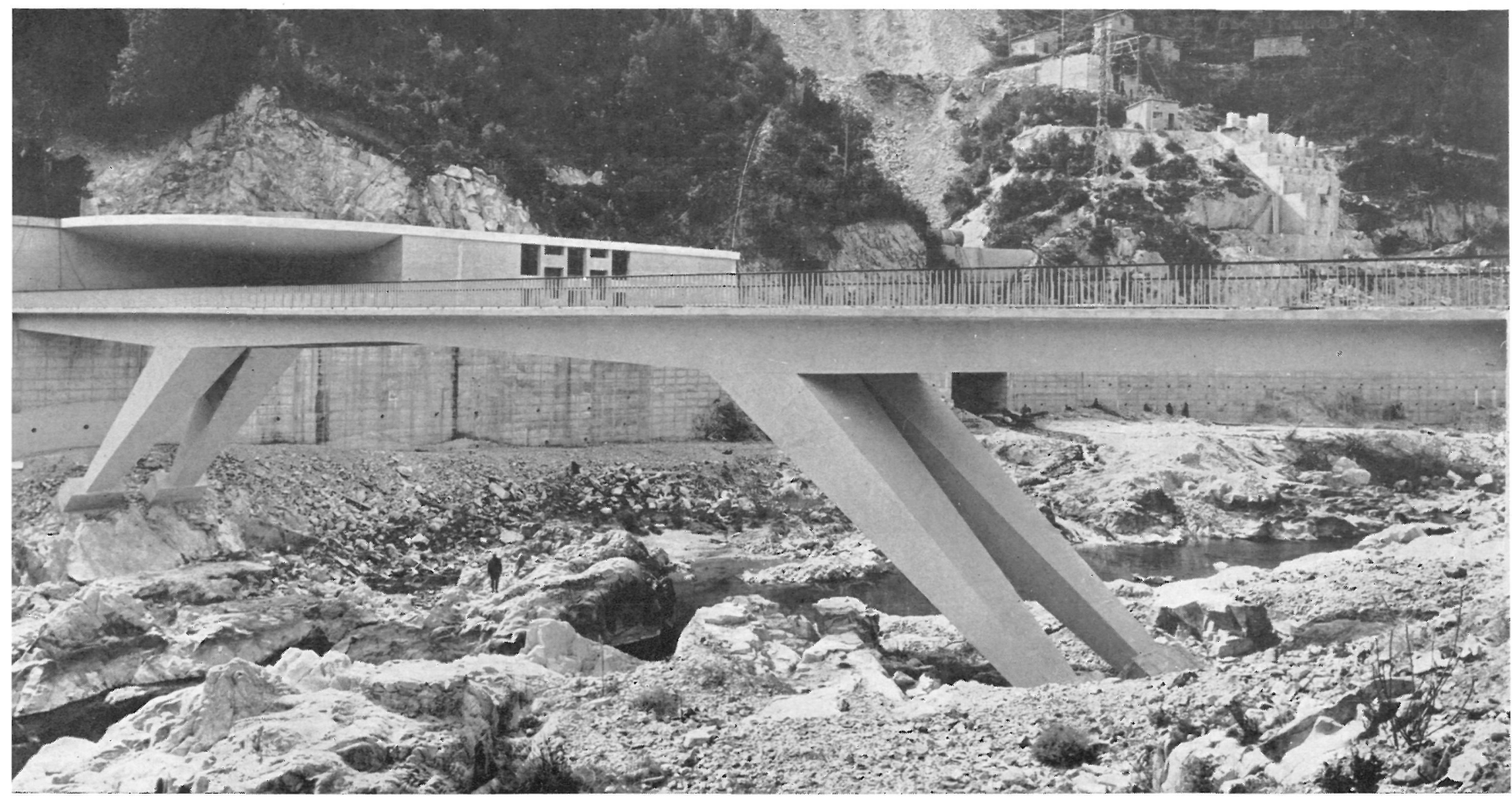

Fotos: BRANGUII

Si añadimos a estas consideraciones el hecho de que todos los elementos estructurales están formados solamente por planos y que no existen curvas que encarecerían tanto los encofrados como la puesta en obra de la ferralla y hormigón, concluiremos que la gran capacidad resistente de esta estructura ofrece un interés digno de tenerse en cuenta a la hora de valorar y decidir la elección de cualquier otro tipo estructural para desarrollar una función análoga.

\subsection{Posibilidades estéticas}

Sin embargo, para nuestra manera de pensar, dejando aparte la tecnología, donde más posibilidades le vemos a este tipo estructural es en sus líneas; líneas que intuimos podrían jugar con los paisajes abiertos y llanos, y encajar con aquellos más cerrados y abruptos. La geometría tan estricta de esta estructura contrastaría también en las zonas urbanas, y su sensación de ligereza y sencillez, sobre todo en grandes luces, no dudamos añadiría algún motivo agradable para el ciudadano. 


\section{Pont En bétom précontraint sur le tem}

Arturo Rebollo, Dr. Ing. Civil

Construit en 1964, dans le cadre des travaux d'aménagement de la chute de Susqueda (Gérone) entrepris par HIDROELECTRICA DE CATALUÑA, S. A., cet ouvrage donne accès à la centrale souterraine. Il en a permis la construction et le montage, et sert, actuellement, à son exploitation.

La structure, prévue pour des surcharges mobiles de 125 tonnes, est celle d'un portique simple, à palées inclinées, dont les épaisseurs varient linéairement. Le tablier, d'une longueur de 60 mètres, est précontraint de bout en bout, chacune des extrémités comportant une articulation mobile.

En vue de l'excellent résultat obtenu par cette disposition structurale, on est amené à en tripler l'échelle, obtenant ainsi des portées de 120-140 mètres, non seulement parce que ses conditions techniques et économiques le permettent, mais aussi parce qu'il en résulterait des ponts d'une beauté remarquable.

\section{Prestressed comfrete bridge overn the Ter miver}

Arturo Rebollo, Dr. Civil Eng.

This work, constructed in 1964, is the access used for the construction, erection and present exploitation of the underground generating station of Susqueda Dam (Gerona), of HIDROELECTRICA DE CATALUNA, S. A.

The structure, foreseen for mobile overloads of 125 tons, is formed by a simple portal with inclined supports and linera variation in width. The floor, whose length is 60 metres, is prestressed from end to end and is fitted with intermediate hinges.

In view of the excellent result of this structural layout, there is a possibility of triplicating its present scale, thus spanning gaps of 120-140 metres, not only because its technical and economic conditions permit it but also because bridges of notable beauty could be built with this type of structure.

\section{Sponmbetrombircke irber dem Ter}

Dipl. Ing. Arturo Rebollo

Dieses Bauwerk, ausgeführt in 1964, dient der Zufahrt zur unterirdischen Zentrale der Staumauer Susqueda (Gerona), HIDROELEGTRICA DE CATALUNA, S. A., und wurde für die Ausführung der Bauarbeiten, Montage der Maschinen und den gegenwärtigen und zukünftigen Betrieb erstellt.

Die Struktur, für eine mobile Ueberlast von 125 Tonnen vorgesehen, besteht aus einem einfachen Rahmen, über schrägen Pfeilern, mit linear veränderlichen Höhen. Die Fahrbahntafel, die 60 Meter lang ist, wurde über die gesamte Länge vorgespannt mit beweglichen Gelenken an beiden Enden.

In Anbetracht der ausgezeichneten Ergebnisse dieser Struktur, kann man darauf schliessen, dass sich die Masse bis auf das dreifache ausdehnen lassen, wobei Lichtweiten von 120-140 Meter überspannt werden können, nicht nur, weil es die technischen und finanziellen Bedingungen erlauben, sondern weil sich auf diese Weise auch Brücken mit besonders schöner Linienführung erstellen lassen. 